\title{
Cluster headache as a first manifestation of multiple sclerosis: case report and literature review
}

\author{
This article was published in the following Dove Press journal: \\ Neuropsychiatric Disease and Treatment \\ 25 November 2014 \\ Number of times this article has been viewed
}

\author{
Milija D Mijajlović* \\ Vuk M Aleksić* \\ Nadežda M Čovičković \\ Šternić \\ Department for Cerebrovascular \\ Disorders and Headaches, Neurology \\ Clinic, Clinical Center of Serbia, \\ School of Medicine, University \\ of Belgrade, Belgrade, Serbia \\ *These authors contributed equally \\ to this work
}

Correspondence: Milija D Mijajlović Neurology Clinic, Clinical Center of Serbia, School of Medicine, University of Belgrade, Dr Subotića 6, II 1000 Belgrade, Serbia

Tel +38I 64 I92 940।

$\mathrm{Fax}+38$ I I| 2684577

Email milijamijajlovic@yahoo.com

\begin{abstract}
Cluster headache $(\mathrm{CH})$ is estimated to be the most common primary trigeminal autonomic headache, although it is a rare disabling medical condition. Dominant symptoms of $\mathrm{CH}$ include severe unilateral orbital, supraorbital, and/or temporal pain, lasting from 15 to 180 minutes if untreated, associated with at least one of various autonomic symptoms during the headache, such as conjunctival injection, lacrimation, nasal congestion and rhinorrhea, facial sweating, miosis, ptosis, and eyelid edema. Headache is not frequently a symptom of multiple sclerosis (MS). The most commonly reported primary headaches are migraine without aura and a tension-type headache. Several described cases involved complicated migraine, ophthalmoplegic migraine-like headache, and finally cluster-like headache. We present a case of a 45-year-old male patient who had typical $\mathrm{CH}$ attacks as the initial and only clinical manifestation of MS, which was diagnosed after cerebrospinal fluid (CSF) isoelectric focusing and brain magnetic resonance imaging (MRI) investigation. He presented as a typical cluster-like headache patient since in the background of the $\mathrm{CH}$ symptoms and signs, were MS demyelinating lesions. In a patient with $\mathrm{CH}$ symptoms one should always think about the possibility of cluster-like-headache, which presents the $\mathrm{CH}$ patient with different underlying diseases, so we proposed a protocol to evaluate such patients and exclude diseases that could be in the background of $\mathrm{CH}$ symptoms.
\end{abstract}

Keywords: demyelinating disease, headache, trigeminal autonomic cephalalgia, diagnosis

\section{Introduction}

Cluster headache $(\mathrm{CH})$ is the most common primary trigeminal autonomic cephalalgia (TAC), although it is a rare, disabling medical condition. Prevalence of $\mathrm{CH}$ in the general population is about $0.1 \%{ }^{1}$ Symptoms of $\mathrm{CH}$ include severe unilateral orbital, supraorbital and/or temporal pain lasting from 15 minutes to 180 minutes if untreated, associated with at least one of the autonomic symptoms, such as conjunctival injection, lacrimation, nasal congestion and rhinorrhea, facial sweating, miosis, ptosis, and eyelid edema. ${ }^{2}$

During the active phase of disease, so-called "cluster periods", attacks are mainly during night, repeating from once every second day to eight times per day. $\mathrm{CH}$ disease evolution can be chronic or episodic. When disease duration exceeds 1 year or when the remission period lasts less than 2 weeks, the chronic form is diagnosed. Some characteristics of $\mathrm{CH}$ are: male preponderance; precipitation of attacks by ingestion of alcohol, nitroglycerin, or histamine; cigarette smoking; head trauma; and even stressful work conditions.,

Although, headache is not generally considered as a symptom of multiple sclerosis (MS), a few studies have investigated the association between these two diseases, and the most recent data show that headache is about 50\% more frequent in MS patients 
than in controls or the general population. ${ }^{5}$ However, the incidence of headache in MS patients varies greatly, from $4 \%$ to $61.8 \%$. These inconsistent data may be explained by different study designs and/or patient's inclusion criteria. ${ }^{5-8}$ The most frequently reported primary headaches are migraine without aura and tension-type headache. Several described cases involved complicated migraine, ophthalmoplegic migraine-like headache, and finally cluster-like headache $(\mathrm{CLH}) .^{5}$

Neuroimaging in $\mathrm{CH}$ patients is usually normal. However, different intracranial pathological changes, such as tumors, aneurysms, arteriovenous malformations, injuries, and infections can be found in $3 \%-5 \%$ of $\mathrm{CH}$ patients. ${ }^{9}$ In some cases, the term CLH is used because the characteristics of the headache pain do not always completely fulfill the International Classification of Headache Disorders (ICHD-II) diagnostic criteria for episodic or chronic $\mathrm{CH} .{ }^{2,9-11}$ However, even in patients fulfilling all proposed diagnostic criteria, intracranial lesions may be discovered. On the other hand, some physicians find the diagnostic criteria to be crucial. This is our opinion also. Therefore, CLH or the sometimes used synonym "secondary cluster headache" only considers cases that completely fulfill the ICHD-II diagnostic criteria of $\mathrm{CH}$ differing only by the presence of another intracranial pathology, which may be involved; further, treatment of the associated disease should cure CLH. Gentile et al have proposed diagnostic criteria for CLH, which are summarized in Table $1 .{ }^{9}$

\section{Case report}

We present a 45-year-old male patient admitted to the Neurology Clinic of the Clinical Center of Serbia, with stabbing pain localized in deep in the left eye, spreading to the left side of the head, followed by conjunctival hyperemia of the left eye, lacrimation, and nasal congestion with rhinorrhea, also on the left side. The pain recurred from three to five times per day, usually lasting about 60 minutes. The pain was very intense, and therapy with several nonsteroidal anti-inflammatory drugs was ineffective. The patient suffered these headaches

\section{Table I Diagnostic criteria for CLH}

International Headache Society criteria for cluster headache are met Physical examination during and outside attacks is normal A lesion is located in a site where trigeminovascular system can be activated

Headache is cured after treatment of the second disease without recurrence

Abbreviation: CLH, cluster-like headache. for 7 days prior to being neurologically investigated (for the first time), at the neurological emergency department of our hospital. The described cluster episode was the first one in patient's life. In the emergency neurological department where the patient was initially examined, we performed brain computed tomography (CT), which revealed normal findings. The patient was treated with oxygen therapy, $15 \mathrm{~L} / \mathrm{min}$ by facial mask for 15 minutes, and the headache almost completely disappeared - he just felt mild numbness around the left eye. The patient was without pain for about 12 hours, but headache attacks appeared again. Clinical and neurological examinations were completely normal. On the second day of hospitalization, we performed magnetic resonance imaging (MRI) of the head, which showed multiple focal demyelinating changes, up to $11 \mathrm{~mm}$ in diameter, localized in the supratentorial frontotemporoparietal white matter, supra- and periventricular corners of the lateral chambers on both sides, in the centrum semiovale, corpus callosum, basal ganglia, and in the left perithalamic region. Also, lesions were found dorsolaterally in the right half of the pons, and in the left middle cerebellar peduncle (brachium pontis). These changes, by their localizations and MRI characteristics, were defined by the neuroradiologist to be typical for MS (Figure 1).

The results of cerebrospinal fluid (CSF) isoelectric focusing after lumbar puncture were positive for oligoclonal bands in the gamma regions, while cytological, biochemical, serological, and virological findings of the CSF were normal.

We also performed additional tests: neuroophtalmological examination, craniogram with paranasal sinuses projection, and digital subtraction angiography. All findings were normal. Complete blood count, biochemical and immunoserological analyses were also within normal range. From the neurophysiological examinations of our patient, the blink reflex study on the left showed findings supporting lesion of the medulla oblongata. Also, brainstem auditory evoked potentials (BAEPs) on the left side showed prolonged latency of III, IV, and V waves, which indicated damage of the upper part of the mesencephalon and pons.

The patient was treated with methylprednisolone $1,000 \mathrm{mg}$ per day intravenously (IV) for 5 consecutive days, with tapering during 12 days. This therapy was introduced as a transitional therapy for frequent $\mathrm{CH}$; after that, we introduced prophylactic therapy for $\mathrm{CH}$, verapamil with slowly increasing dose to the target stable dose of $320 \mathrm{mg}$ per day, which he took regularly for 1 year. The headache attacks gradually subsided within the first 5 days, and the patient was headache-free during 3 years of follow up, with 


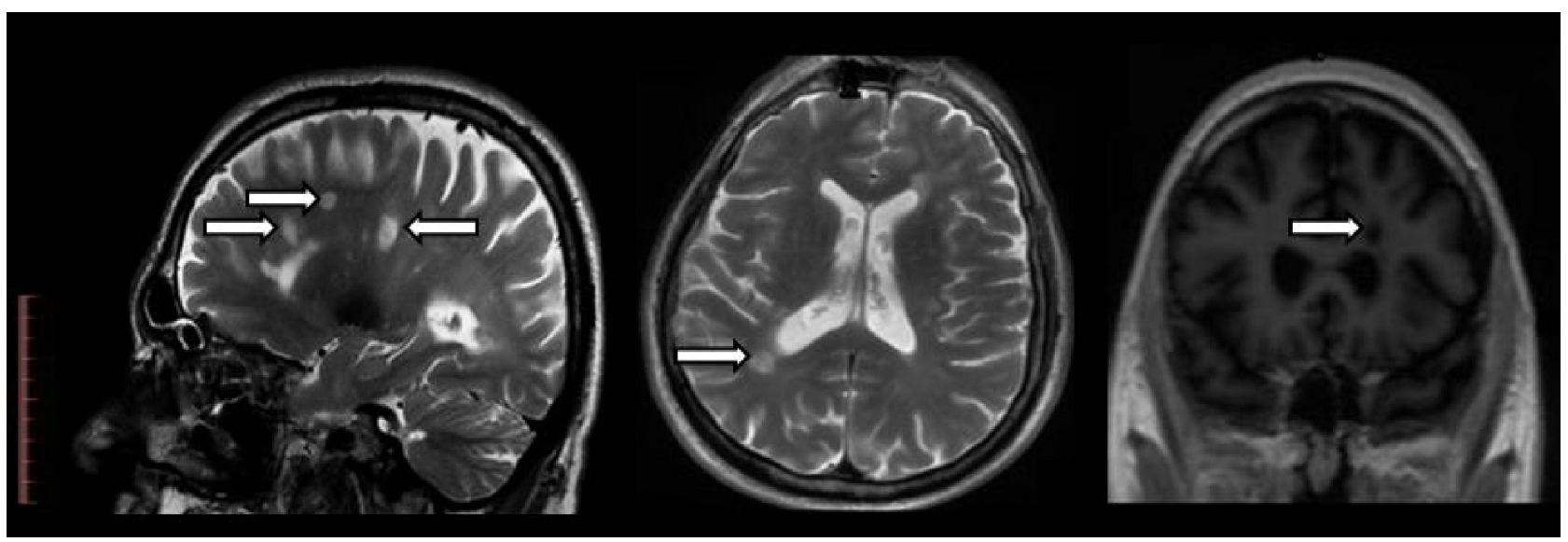

Figure I MRI sections showing multiple demyelinating brain lesions (arrows).

Notes: MRI scans of our presented case, showing multiple focal demyelinating changes, up to II mm in diameter: hyperintense in T2w (sagittal section, left image) and FLAIR (axial section, middle image), isointense in T2w (coronal section, right image) localized in the supratentorial frontotemporoparietal white matter, supra- and periventricular corners of the lateral chambers on both sides, in the centrum semiovale, corpus callosum, basal ganglia, and in the left perithalamic region. Also, lesions were found dorsolaterally in the right side of pons, and in the left middle cerebellar peduncle.

Abbreviations: FLAIR, fluid attenuated inversion recovery; MRI, magnetic resonance imaging; T2w, T2-weighted.

normal neurological findings and with no MS relapse, either clinically or neuroradiologically.

\section{Discussion and literature review}

Our patient is a typical CLH patient. This rare type of headache was the first and only symptom of MS in our patient. The patient was diagnosed as having MS according to the new McDonald's criteria (2011 revision). ${ }^{12}$ The present case raises a few questions, and the first one is: Is there a relationship between the two pathological conditions, or is the association just a coincidence? We think that relation between these two diseases exists and that coincidence is less probable. Our patient had multiple demyelinating lesions, including brain stem localization, and according to the literature, several times $\mathrm{CH}$ has been found in patients with similar distribution of demyelination. ${ }^{13,14}$ A similar case was described by Leandri et al. ${ }^{13}$ The description adheres exactly to the ICHD-II criteria, and preventive therapy was also effective. A demyelination lesion was diagnosed, by MRI, in the pons at the trigeminal nerve root entry zone on the side of the headache. In this situation, one should expect trigeminal neuralgia pain; however, the pain was typical for $\mathrm{CH}$. The authors stated that there was probable interaction between the demyelinating lesion and trigeminovascular system, which caused altered activity of the fifth cranial nerve. ${ }^{13}$ A similar explanation can be given in our case.

Many other pathological conditions have been connected with CLH pain. West and Todman reported a case of chronic CLH associated with a vertebral artery aneurysm. They presented a case of chronic CLH lasting for 12 years.
The patient had an aneurysm at the junction of the right vertebral and right posterior inferior cerebellar arteries, and after its surgical removal, the $\mathrm{CH}$ symptoms completely resolved. These two pathological conditions were on the same side, suggesting a possible relationship. ${ }^{15}$ Hannerz presented a patient with parasellar meningioma under the guise of $\mathrm{CH}$. Removal of the tumor caused complete amelioration, making the hypothesis of relationship between the lesions in the cavernous sinus region and the $\mathrm{CH}$ symptoms and signs more likely. ${ }^{16}$ Also, subclavian steal phenomenon was reported, by Piovesan et al as a cause of CLH. The subclavian steal phenomenon, due to a thrombosed subclavian artery, and the $\mathrm{CH}$ pain were on the same side, but more interestingly, bypass surgery with a saphenous vein normalized blood circulation, and a few years later, there were no signs of CLH. The authors proposed the interesting theory that increased blood flow in the internal carotid arteries provoked venous enlargement in the cavernous sinus, which led to CLH. ${ }^{17}$ Also, some authors have described association between different tumors and $\mathrm{CH}$, but most of them did not fulfill ICHD-II criteria. ${ }^{2}$ Domitrz et al conducted a MEDLINE $^{\circledR}$ research study to identify all published CLH or symptomatic CH studies, between 1980 and 2001. They had problems in detecting all CLH cases as some were published in $\mathrm{CH}$ series. The authors identified 68 cases, but because of many investigation difficulties, some cases were probably overlooked. They classed all cases in four groups, based on the second neurological disease: 1) facial diseases; 2) cavernous sinus region pathologies; 3) cranial vessels; and 4) other diseases, including MS. ${ }^{2}$ These data 
suggest caution in patients with symptoms of $\mathrm{CH}$, and we recommend complete neuroradiological assessment of such patients, because a life-threatening pathology can be hidden in the background.

TACs belong to the primary headache syndromes. They can be easily recognized by unilateral high-intensity pain, headaches of short duration associated with paroxysmal ipsilateral facial autonomic symptoms. This headache group includes three entities: $\mathrm{CH}$; paroxysmal hemicrania; and short-lasting unilateral pain with characteristics of neuralgiform headache, followed by conjunctival hyperemia and lacrimation. Reviewing 31 cases of patients with TAC, Favier et al found that even TACs with typical presentation can be caused by an underlying lesion, but unfortunately, the clinical warning symptoms and signs of lesion are relatively rare, thus the authors recommended complete neuroradiological investigation in all TAC patients. ${ }^{18}$ This is in accordance with our practical clinical suggestion for such patients.

Edvardsson described the case of a 24-year-old patient who presented with a 1-month history of pain located in the left orbit. He was diagnosed with $\mathrm{CH}$. The patient responded to symptomatic treatment, but 2 weeks later, CT scan showed signs of acute maxillary sinusitis on the side of the headache.
The headache attacks resolved completely after appropriate treatment with antibiotics and sinus puncture. The author concluded that:

[...] neuroimaging, preferably contrast-enhanced magnetic resonance imaging, including sinuses examination, should always be considered in patients with $\mathrm{CH}$ despite normal findings of neurological examination. ${ }^{19}$

On the other hand, only few months later, Edvardsson conducted a study of $63 \mathrm{CH}$ or CLH cases from the literature, which were associated with an underlying intracranial lesion. He suggested that:

[...] neuroimaging, preferably contrast-enhanced magnetic resonance imaging/magnetic resonance angiography, should be undertaken only in patients with atypical symptomatology, late onset, abnormal examination findings (including Horner's syndrome), or those resistant to the appropriate medical treatment. ${ }^{20}$

However, we think that lack of prospective studies, which are needed to identify the prevalence of symptomatic $\mathrm{CHs}$, imposes an obligation to conduct full neuroradiological assessment in patients with such symptoms.

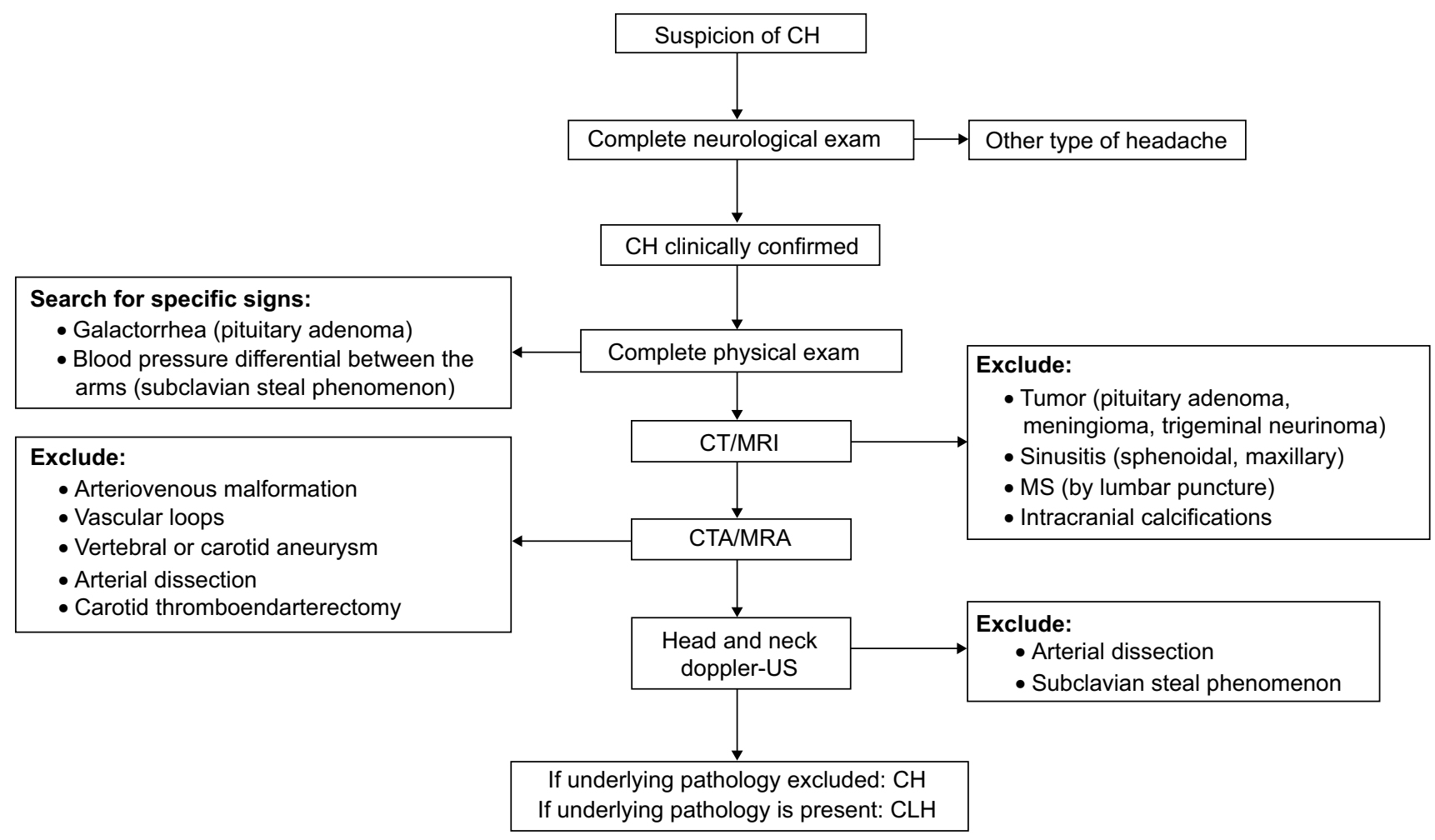

Figure 2 Protocol for investigation of $\mathrm{CH}$ patients.

Abbreviations: $\mathrm{CH}$, cluster headache; $\mathrm{CLH}$, cluster-like headache; $\mathrm{CT}$, computed tomography; CTA, computed tomography angiography; MRI, magnetic resonance imaging; MRA, magnetic resonance angiography; MS, multiple sclerosis; US, ultrasound. 
Another detail should be mentioned. It is well known that $\mathrm{CH}$ onset usually occurs between the third and fifth decade. ${ }^{20,21}$ The peak incidence for both sexes is reached between age 20-29 years. ${ }^{21,22}$ In the Edvardsson's review of symptomatic cases, the mean age of symptom presentation was 40 years, which supports the opinion that late onset $\mathrm{CH}$ should prompt careful evaluation. ${ }^{20}$ Our patient was 45 years at admission, and this was one of the warning signs that he should be given extra attention.

One of the quite neglected points is the quality of neurological, physical, and clinical examinations in patients with $\mathrm{CH}$ symptoms. In fact, a few cases prove that, even in patients that fulfill the ICHD criteria for $\mathrm{CH}$, the examination was not ordinary. ${ }^{2,23,24}$ Physical findings should be as complete as possible and not limited to the face and cranial nerve explorations. A complete physical and, of course, neurological examination is always advisable because some indirect signs of a lesion, such as galactorrhea, for pituitary adenomas, or blood pressure difference between the arms, in subclavian steal phenomenon, may be overlooked without thorough examination. ${ }^{2}$ Mainardi et al also emphasized the importance of accurate, clinical evaluation in patients with $\mathrm{CH}$ symptoms. ${ }^{25}$ Although our patient had many demyelinating lesions in different brain areas, the neurological and clinical findings were unremarkable, except for the specific signs of CLH.

\section{Conclusion}

We presented a 45-year-old male patient who had typical $\mathrm{CH}$ symptoms as a first manifestation of MS, which was diagnosed after CSF isoelectric focusing and MRI investigation. He was a typical CLH patient since in the background of the $\mathrm{CH}$ symptoms and signs, were MS demyelinating lesions. $\mathrm{CH}$ attacks as clinical overture symptoms of MS have been considered to be rare, until recently, but a growing number of these cases have been described in the last few years. However, the association between these two pathological conditions is unclear. On the other hand, many other life-threatening diseases can be the probable cause of $\mathrm{CH}$. Knowing the abovementioned facts, we created a protocol for patients with $\mathrm{CH}$ symptoms and signs. This protocol is based on possible underlying pathology that can be seen as part of CLH. The diseases for consideration have all already been described and reported in the literature (Figure 2). 2,15-17,26-36

\section{Disclosure}

The authors report no conflicts of interest in this work.

\section{References}

1. Goadsby PJ. Trigeminal autonomic cephalalgias (TACs). Acta Neurol Belg. 2001;101:10-19.

2. Giraud P, Jouanneau E, Borson-Chazot F, Lanteri-Minet M, Chazot G. Cluster-like headache: literature review. J Headache Pain. 2002; 3(2):71-78.

3. Manzoni GC. Cluster headache and lifestyle: remarks on a population of 374 male patients. Cephalalgia. 1999;19(2):88-94.

4. May A, Bahra A, Büchel C, Frackowiak RS, Goadsby PJ. Hypothalamic activation in cluster headache attacks. Lancet. 1998;352(9124): 275-278.

5. La Mantia L. Headache and multiple sclerosis: clinical and therapeutic correlations. Neurol Sci. 2009;30 Suppl 1:S23-S26.

6. D’Amico D, La Mantia L, Rigamonti A, et al. Prevalence of primary headaches in people with multiple sclerosis. Cephalalgia. 2004;24(11): 980-984.

7. Freedman MS, Gray TA. Vascular headache: a presenting symptom of multiple sclerosis. Can J Neurol Sci. 1989;16(1):63-66.

8. Villani V, Prosperini L, Ciuffoli A, et al. Primary headache and multiple sclerosis: preliminary results of a prospective study. Neurol Sci. 2008; 29 Suppl 1:S146-S148.

9. Gentile S, Ferrero M, Vaula G, Rainero I, Pinessi L. Cluster headache attacks and multiple sclerosis. J Headache Pain. 2007;8(4): 245-247.

10. Headache Classification Subcommittee of the International Headache Society. The International Classification of Headache Disorders: 2nd edition. Cephalalgia. 2004;24 Suppl 1:S9-S160.

11. Domitrz I, Gawel M, Maj E. Cluster headache - a symptom of different problems or a primary form? A case report. Neurol Neurochir Pol. 2013;47(2):184-188.

12. Polman CH, Reingold SC, Banwell B, et al. Diagnostic criteria for multiple sclerosis: 2010 revisions to the McDonald criteria. Ann Neurol. 2011;69(2):292-302.

13. Leandri M, Cruccu G, Gottlieb A. Cluster headache-like pain in multiple sclerosis. Cephalalgia. 1999;19(8):732-734.

14. Donat J. A patient with cluster headache - due to a brainstem lesion. Headache. 2012;52(6):1035-1036.

15. West $\mathrm{P}$, Todman $\mathrm{D}$. Chronic cluster headache associated with a vertebral artery aneurysm. Headache. 1991;31(4):210-212.

16. Hannerz J. A case of parasellar meningioma mimicking cluster headache. Cephalalgia. 1989;9(4):265-269.

17. Piovesan EJ, Lange MC, Werneck LC, et al. Cluster-like headache. A case secondary to the subclavian steal phenomenon. Cephalalgia. 2001; 21(8):850-851.

18. Favier I, van Vliet JA, Roon KI, et al. Trigeminal autonomic cephalgias due to structural lesions: a review of 31 cases. Arch Neurol. 2007; 64(1):25-31.

19. Edvardsson B. Cluster headache associated with acute maxillary sinusitis. Springerplus. 2013;2:509.

20. Edvardsson B. Symptomatic cluster headache: a review of 63 cases. Springerplus. 2014;3:64.

21. Nesbitt AD, Goadsby PJ. Cluster headache. BMJ. 2012;344:e2407.

22. Ekbom K, Svensson DA, Träff H, Waldenlind E. Age at onset and sex ratio in cluster headache: observations over three decades. Cephalalgia. 2002;22(2):94-100.

23. Greve E, Mai J. Cluster headache-like headaches: a symptomatic feature? A report of three patients with intracranial pathologic findings. Cephalalgia. 1988;8(2):79-82.

24. Milos P, Havelius U, Hindfelt B. Clusterlike headache in a patient with pituitary adenoma. With review of the literature. Headache. 1996;36(2):184-188.

25. Mainardi F, Trucco M, Maggioni F, Palestini C, Dainese F, Zanchin G. Cluster-like headache. A comprehensive reappraisal. Cephalalgia. 2010;30(4):399-412.

26. Tfelt-Hansen P, Paulson OB, Krabbe AA. Invasive adenoma of the pituitary gland and chronic migrainous neuralgia. A rare coincidence or a causal relationship? Cephalalgia. 1982;2(1):25-28. 
27. Lefevre JP, Simmat G, Bataille B, et al. [Cluster headache due to meningioma. 2 cases]. Presse Med. 1984;13(38):2323. French.

28. Masson C, Lehericy S, Guillaume B, Masson M. Cluster-like headache in a patient with a trigeminal neurinoma. Headache. 1995;35(1): 48-49.

29. Takeshima T, Nishikawa S, Takahashi K. Cluster headache like symptoms due to sinusitis: evidence for neuronal pathogenesis of cluster headache syndrome. Headache. 1988;28(3):207-208.

30. Burrow GN, Wortzman G, Rewcastle NB, Holgate RC, Kovacs K. Microadenomas of the pituitary and abnormal sellar tomograms in an unselected autopsy series. N Engl J Med. 1981;304(3):156-158.

31. Testa D, Frediani F, Bussone G. Cluster headache-like syndrome due to arteriovenous malformation. Headache. 1988;28(1):36-38.
32. Hindfelt B, Olivecrona $\mathrm{H}$. Cerebral arteriovenous malformation and cluster-like headache. Headache. 1991;31(8):514-517.

33. Gawel MJ, Willinsky RA, Krajewski A. Reversal of cluster headache side following treatment of arteriovenous malformation. Headache. 1989;29(7):453-454.

34. Cremer PD, Halmagyi GM, Goadsby PJ. Secondary cluster headache responsive to sumatriptan. J Neurol Neurosurg Psychiatry. 1995;59(6):633-634.

35. Björne A, Hindfelt B, Havelius U. Recurrence of cluster headache after carotid thrombendarterectomy. Headache. 1994;34(4):230-233.

36. Narbone MC, D'Amico D, Di Maria F, Arena MG, Longo M. Clusterlike headache and a median intracranial calcified lesion: case report. Headache. 1991;31(10):684-685.

\section{Publish your work in this journal}

Neuropsychiatric Disease and Treatment is an international, peerreviewed journal of clinical therapeutics and pharmacology focusing on concise rapid reporting of clinical or pre-clinical studies on a range of neuropsychiatric and neurological disorders. This journal is indexed on PubMed Central, the 'PsycINFO' database and CAS, and is the official journal of The International Neuropsychiatric Association (INA). The manuscript management system is completely online and includes a very quick and fair peer-review system, which is all easy to use. Visit http://www.dovepress.com/testimonials.php to read real quotes from published authors.

\footnotetext{
Submit your manuscript here: http://www.dovepress.com/neuropsychiatric-disease-and-treatment-journal
} 